\title{
Global Inward Foreign Direct Investment: Trends and Concentration Patterns
}

\author{
K V Bhanu Murthy* and Manoj Kumar Sinha**
}

\begin{abstract}
With globalisation, there has been a phenomenal rise in Foreign Direct Investment (FDI). This has led to an international relocation of production and consequent generation of income and employment. This, in turn, has led to international specialisation of production and is likely to maximize global welfare. This could happen only if the concentration of FDI is low or FDI is well distributed. We use a set of new indices and Herfindahl index of concentration to examine concentration of inward FDI in terms of flow and stock during 1990-2009. The results clearly indicate that there is a low concentration in distribution of FDI in terms of inflow and stock. The low concentration ratio implies that the share of developing countries is increasing. It implies that the distribution of FDI has been moving towards uniform distribution of productive resources across countries, which is the basic purpose of globalisation and competition.
\end{abstract}

Keywords: Foreign Direct Investment, Gross Domestic Product, Growth Rate.

\subsection{Introduction}

As has been established the international patterns of FDI are governed by the new economic order based on free flow of capital along with technology and managerial resources. The process of globalisation has aided such international FDI flows. It is important that capital flows in the form of FDI consistently flow to the most desirable regions which have unutilised resources of the world. These regions need to receive an equitable distribution of FDI. Hence, these countries should receive FDI in such a manner so as to ensure optimum allocation of resources. This could happen only if the concentration of FDI is low or FDI is well distributed. It is therefore apparent that there is a strong relationship between international FDI and concentration pattern.

*Professor, Department of Commerce, Delhi School of Economics, University of Delhi.

${ }^{* *}$ Assistant Professor, PGDAV College, University of Delhi. 
2 |MUDRA: Journal of Finance and Accounting, Vol. 1, Issue 2

The expectation is that the international FDI would be uniformly distributed to enable a rational distribution of resources. It implies that the concentration of FDI should be low amongst the recipient (host) countries.

\subsection{Data and Methodology}

\subsection{Hypotheses}

We test the following three hypotheses in our study.

1. Concentration pattern of inward FDI has not changed.

2. Concentration pattern of inward FDI has not changed in developed countries.

3. Concentration pattern of inward FDI has not changed in developing countries.

\subsection{Data source}

We have collected online FDI and GDP data for our study mainly from UNCTAD (United Nations Conference on Trade and Development). We have constructed a data set of 53 common countries include developed and developing countries for FDI for periods 1990-2009 in terms of inflows, inward stock, outflows and outward stock.

\subsection{Research methodology}

\section{Three Level Indices}

The purpose of three levels of indices is to expose what is the relative position of each country with respect to average both in terms of FDI and GDP. Further, it tells us the change in relative position over time with the help of temporal indices. The overall change is measured by average across the countries. We have given a set of formalised and stylized indices at four point of time for FDI and GDP across centres. These indices are based on Jha and Bhanu Murthy, et al. (1999). There are two set of indices at the first level- one for FDI and another for GDP.

First level of indices- is based on the ratio of FDI and GDP in a particular centre with respect to national average of FDI and GDP respectively.

Relative Index of FDI

$$
\boldsymbol{F}_{r f}^{c}=\frac{f_{t}^{c}}{\left[\sum_{c=1}^{n} f_{t}^{c} / n\right]}
$$

Where, $\mathrm{t}=$ 1990, 1995, 2000, 2005 and 2009 .

$$
\begin{aligned}
& \mathrm{c}=1,2, \ldots \ldots \ldots . .53 \\
& \mathrm{n}=53 \\
& \boldsymbol{F}_{\boldsymbol{r} f}^{\boldsymbol{c}}=\text { FDI ratio of } \boldsymbol{c}^{\boldsymbol{t h}} \text { country with respect to average FDI in } \boldsymbol{t}^{\boldsymbol{t h}} \text { period. }
\end{aligned}
$$


Relative Index of GDP

$$
P_{r f}^{c}=\frac{p_{t}^{c}}{\left[\sum_{c=1}^{n} p_{t}^{c} / n\right]}
$$

$\boldsymbol{P}_{\boldsymbol{r} f}^{\boldsymbol{c}}=$ GDP ratio of $\boldsymbol{c}^{\boldsymbol{t h}}$ Country with respect to average GDP in $\boldsymbol{t}^{\boldsymbol{t h}}$ period.

Second level of Indices - is a temporal ratio of first ratio at two point of time for both FDI and GDP

\section{Temporal Index of FDI}

$$
\boldsymbol{R} \boldsymbol{F}_{t \prime \boldsymbol{t}}^{\boldsymbol{c}}=\frac{\boldsymbol{F}_{r t \prime}^{\boldsymbol{c}}}{\boldsymbol{F}_{r t}^{c}} ; \text { where } \boldsymbol{R} \boldsymbol{F}_{\boldsymbol{t} / \boldsymbol{t}}^{\boldsymbol{c}}=\text { Temporal Index of FDI. }
$$

This measures change in relative position of the FDI index of a particular country over between two periods of time. This yields three indices between 1995 and 1990; between 2000 and 1995; between 2005 and 2000 and between 2009 and 2005.

Average Ratio of Relative FDI

$$
A R F_{T}=\sum_{c=1}^{n} R F_{t}^{c} / n
$$

$\boldsymbol{A} \boldsymbol{R} \boldsymbol{F}_{\boldsymbol{T}}=$ Average of ratio of relative FDI between two points of time

Change in FDI average

$$
A R F_{T^{\prime} T}=\frac{A R F_{T^{\prime}}}{A R F_{T}}
$$

$\boldsymbol{A R} \boldsymbol{F}_{\boldsymbol{T}^{\prime} \boldsymbol{T}}=$ Change in average between two points of time.

Temporal Index of GDP

$$
\boldsymbol{R} \boldsymbol{P}_{\boldsymbol{t} / \boldsymbol{t}}^{\boldsymbol{c}}=\frac{\boldsymbol{P}_{r t \prime}^{\boldsymbol{c}}}{\boldsymbol{P}_{r t}^{c}} ; \text { where } \boldsymbol{R} \boldsymbol{P}_{\boldsymbol{t} t \boldsymbol{t}}^{\boldsymbol{c}}=\text { Temporal Index of GDP. }
$$

This is index of the ratio between GDP indices at two points of time. This measures change in relative position of the FDI index of a particular country between two points of time. This yields three indices between 1995 and 1990; between 2000 and 1995; between 2005 and 2000 and between 2009 and 2005.

Average Ratio of Relative GDP

$$
\boldsymbol{A R P} \boldsymbol{P}_{T}=\sum_{c=1}^{n} \boldsymbol{R P}_{t}^{c} / n
$$

$\boldsymbol{A} \boldsymbol{R} \boldsymbol{P}_{\boldsymbol{T}}=$ Average of ratio of relative GDP between two points of time 
4 MUDRA: Journal of Finance and Accounting, Vol. 1, Issue 2

Change in GDP average

$A R P_{T^{\prime} T}=\frac{A R P_{T \prime}}{A R P_{T}}$

$\boldsymbol{A R} \boldsymbol{P}_{\boldsymbol{T}^{\prime} \boldsymbol{T}}=$ Change in average between two points of time.

Third level of Indices - is ratio between the temporal indices of FDI and corresponding temporal indices of GDP.

$$
\begin{aligned}
& R R_{t}^{c}=\frac{R F_{t \prime t}^{c}}{R P_{t \prime t}^{c}} \\
& A R R_{T}=\sum_{c=1}^{n} R R_{t}^{c} / n \\
& A R R_{T^{\prime} T}=\frac{A R R_{T^{\prime}}}{A R R_{T}}
\end{aligned}
$$

Power of these indices lies in exposing what is the relative position of each country with respect to average both in terms of FDI and GDP. Also it tells us the change in relative position over time with the help of temporal indices. Overall change is measured by average across the countries.

\section{Herfindahl-Hirschman Index of Concentration}

Herfindahl-Hirschman Index (HHI) is a commonly accepted measure of market concentration. It is calculated by squaring the market share of each FDI recipient country in a market, and then summing the resulting number (Bhanu Murthy and Deb, 2008). The HHI is expressed as:

$$
H H I=\sum_{i=1}^{N} S_{i}^{2}
$$

where ' $\mathrm{Si}$ ' is the market share of FDI recipient country $\mathrm{i}$ in the market and ' $\mathrm{N}$ ' is the number of counties. This index is range from $1 / \mathrm{N}$ to one, where ' $\mathrm{N}$ ' is the number of countries.

A HHI index below 0.01 indicates a highly competitive.

A HHI index below 0.1 indicates not concentrated.

A HHI index between 0.1 to 0.18 indicates low concentration.

A HHI index above 0.18 to 0.30 indicates moderate concentration.

A HHI index above 0.30 indicates high concentration.

In absolute terms, we have already seen that concentration of FDI inflow and stock has been consistently declined over periods. Due to globalisation and global competitive environment, global productive resources are redistributed on the basis of efficiency. This leads to optimum utilisation of resources. 


\subsection{Literature Review}

Stallings (2007) analyses the benefits and costs of financial globalisation. While most attention has been placed on the opportunity to obtain additional capital, the benefits from incorporating international norms are also highlighted. The article examines the trends in capital flows, both from public sector institutions and private investors, placing special emphasis on foreign direct investment and remittances. Major problems identified are the skewed distribution of foreign investment not only among regions and countries, but also among types of firms as well as its volatility. The article concludes with a set of policy recommendations to spread the benefits of foreign capital and to make it more productive.

Banga (2006) estimates the impact of bilateral investment agreements (BITs) on FDI inflows into fifteen Asian developing countries for the period 1980-81 to 1999-2000 and examines whether signing an investment agreement with a developed country or a developing country matters. It also examines the impact of regional investment agreements, namely between APEC and ASEAN countries on FDI inflows. Panel data estimations are undertaken and the results show that signing BITs attracts FDI inflows. However, it is BITs with developed countries that increase FDI inflows as compared to BITs with developing countries. Results indicate that investment agreement between APEC countries has increased FDI inflows but that amongst ASEAN countries has had no impact.

Goldar and Ishigami (1999): This paper analyses trends in FDI in Asia, with a special focus on FDI flows from Japan. It relates FDI flows to changing industrial structure and to trade flows. An econometric analysis is done to identify key determinants of FDl flows to Asian countries. Japan has been the main source of FDl flows to Asia. Japanese FDI has helped cost reduction and export promotion in the host countries. But, in the process, Japan has created a large trade surplus with these countries.

\subsection{Results and Analysis}

\subsection{Relationship between FDI and GDP}

We have given a set of formalised and stylized indices at five points of time (i.e. 1990, 1995, 2000, 2005 and 2009) for FDI inflows and GDP across countries. These indices are based on Jha and Bhanu Murthy, et al. (1999).

- I level index: Relative Index.

- II level Index: Temporal Index. 
6 | MUDRA: Journal of Finance and Accounting, Vol. 1, Issue 2

III level Index: Ratio of Temporal Index of FDI and GDP.

The aggregate level changes can be understood with the help of average, change in average, standard deviation, coefficient of variation (CV) of the individual indices and ratio of coefficient of variation at four points of time. Further, the aggregate changes can be understood by taking the minimum $\mathrm{CV}$ as the base.

In terms of absolute amount of FDI inflows, the average FDI inflows have been continuously increasing except 2005 and 2009. In 2000, the average FDI inflows have increased more than four times of FDI inflows in 1995. This fluctuation in FDI inflows has also increased standard deviation. This clearly shows that there has been increased in standard deviation with increase in FDI inflows and vice-versa. However, Coefficient of variation $(\mathrm{CV})$ and $\mathrm{CV}$ ratio of FDI inflows have been continuously decreasing except 2000 (Table 1a).

Relative indices of FDI indicate the relative position of FDI inflow in a particular country with respect to average FDI in a particular year. The results do not give any clear trend of FDI inflow in a country with respect to average FDI. This implies that FDI inflows among the nations have been changing over periods due to international competition for FDI. Now we see temporal indices of FDI between two periods. Average of temporal index has been continuously decreasing except 2005/2000. However Standard deviation, $\mathrm{CV}$ and $\mathrm{CV}$ ratio have been continuously decreasing across countries (Table 1a).

Table 1a: Indices of Country-wise Distribution of FDI Inflows

\begin{tabular}{|c|c|c|c|c|c|c|c|c|c|}
\hline \multirow[t]{2}{*}{ Countries } & \multicolumn{5}{|c|}{ FDI - Relative Index } & \multicolumn{4}{|c|}{ FDI - Temporal Index } \\
\hline & 1990 & 1995 & 2000 & 2005 & 2009 & $\begin{array}{r}1995 \\
/ 1990 \\
\end{array}$ & $\begin{array}{l}2000 / \\
1995\end{array}$ & $\begin{array}{l}2005 / \\
2000\end{array}$ & $\begin{array}{l}2009 / \\
2005\end{array}$ \\
\hline Argentina & 0.49 & 0.94 & 0.41 & 0.28 & 0.28 & 1.94 & 0.44 & 0.69 & 0.98 \\
\hline Australia & 2.15 & 2.01 & 0.55 & 1.75 & 1.28 & 0.94 & 0.27 & 3.17 & 0.73 \\
\hline Austria & 0.17 & 0.32 & 0.35 & 0.58 & 0.40 & 1.85 & 1.09 & 1.66 & 0.69 \\
\hline Bahrain & 0.10 & 0.07 & 0.01 & 0.06 & 0.01 & 0.74 & 0.20 & 3.92 & 0.26 \\
\hline $\begin{array}{l}\text { Belgium- } \\
\text { Luxembourg }\end{array}$ & 2.13 & 1.80 & 3.51 & 2.17 & 3.46 & 0.84 & 1.95 & 0.62 & 1.60 \\
\hline Brazil & 0.26 & 0.74 & 1.30 & 0.81 & 1.47 & 2.83 & 1.75 & 0.62 & 1.82 \\
\hline $\begin{array}{l}\text { British Virgin } \\
\text { Islands }\end{array}$ & 0.00 & 0.15 & 0.39 & 0.65 & 1.44 & 30.92 & 2.60 & 1.67 & 2.19 \\
\hline Canada & 2.01 & 1.56 & 2.64 & 1.38 & 1.06 & 0.77 & 1.70 & 0.52 & 0.77 \\
\hline Chile & 0.18 & 0.50 & 0.19 & 0.37 & 0.72 & 2.84 & 0.39 & 1.95 & 1.92 \\
\hline
\end{tabular}


Global Inward Foreign Direct Investment: Trends and Concentration Patterns

\begin{tabular}{|c|c|c|c|c|c|c|c|c|c|}
\hline \multirow[t]{2}{*}{ Countries } & \multicolumn{5}{|c|}{ FDI - Relative Index } & \multicolumn{4}{|c|}{ FDI - Temporal Index } \\
\hline & 1990 & 1995 & 2000 & 2005 & 2009 & \begin{tabular}{|l|}
1995 \\
$/ 1990$ \\
\end{tabular} & $\begin{array}{l}2000 / \\
1995\end{array}$ & $\begin{array}{l}2005 / \\
2000\end{array}$ & $\begin{array}{l}2009 / \\
2005\end{array}$ \\
\hline China & 0.92 & 6.31 & 1.61 & 3.89 & 5.39 & 6.83 & 0.25 & 2.41 & 1.39 \\
\hline $\begin{array}{l}\text { China, Hong } \\
\text { Kong SAR }\end{array}$ & 0.87 & 1.05 & 2.45 & 1.81 & 2.75 & 1.20 & 2.34 & 0.74 & 1.52 \\
\hline $\begin{array}{l}\text { China, Taiwan } \\
\text { Province of }\end{array}$ & 0.35 & 0.26 & 0.19 & 0.09 & 0.16 & 0.74 & 0.74 & 0.45 & 1.82 \\
\hline Colombia & 0.13 & 0.16 & 0.10 & 0.55 & 0.41 & 1.23 & 0.59 & 5.71 & 0.74 \\
\hline Costa Rica & 0.04 & 0.06 & 0.02 & 0.05 & 0.08 & 1.32 & 0.28 & 2.86 & 1.62 \\
\hline Czech Republic & 0.04 & 0.43 & 0.20 & 0.62 & 0.15 & 9.86 & 0.46 & 3.16 & 0.25 \\
\hline Denmark & 0.30 & 0.73 & 0.65 & 0.48 & 0.44 & 2.43 & 0.89 & 0.74 & 0.92 \\
\hline Egypt & 0.19 & 0.10 & 0.05 & 0.29 & 0.38 & 0.51 & 0.49 & 5.91 & 1.32 \\
\hline France & 4.14 & 3.98 & 1.71 & 4.56 & 3.38 & 0.96 & 0.43 & 2.67 & 0.74 \\
\hline Germany & 0.79 & 2.02 & 7.84 & 2.55 & 2.02 & 2.58 & 3.87 & 0.32 & 0.79 \\
\hline Hungary & 0.15 & 0.86 & 0.11 & 0.41 & 0.39 & 5.85 & 0.13 & 3.79 & 0.95 \\
\hline India & 0.06 & 0.36 & 0.14 & 0.41 & 1.96 & 5.77 & 0.39 & 2.88 & 4.81 \\
\hline Ireland & 0.16 & 0.24 & 1.02 & 0.91 & 1.42 & 1.47 & 4.20 & 0.89 & 1.55 \\
\hline Israel & 0.04 & 0.27 & 0.23 & 0.23 & 0.22 & 7.30 & 0.88 & 0.98 & 0.96 \\
\hline Italy & 1.68 & 0.81 & 0.53 & 1.07 & 1.73 & 0.48 & 0.65 & 2.03 & 1.62 \\
\hline Jamaica & 0.05 & 0.02 & 0.02 & 0.04 & 0.06 & 0.54 & 0.75 & 1.98 & 1.64 \\
\hline Japan & 0.46 & 0.01 & 0.33 & 0.15 & 0.68 & 0.02 & 47.13 & 0.45 & 4.54 \\
\hline Lebanon & 0.00 & 0.01 & 0.04 & 0.14 & 0.27 & 3.45 & 6.47 & 3.69 & 1.93 \\
\hline Malaysia & 0.69 & 0.98 & 0.15 & 0.22 & 0.08 & 1.41 & 0.15 & 1.46 & 0.36 \\
\hline Mauritius & 0.01 & 0.00 & 0.01 & 0.00 & 0.01 & 0.29 & 3.48 & 0.20 & 6.54 \\
\hline Mexico & 0.70 & 1.60 & 0.71 & 1.18 & 0.71 & 2.30 & 0.44 & 1.65 & 0.60 \\
\hline Morocco & 0.04 & 0.06 & 0.02 & 0.09 & 0.08 & 1.28 & 0.30 & 5.32 & 0.85 \\
\hline Netherlands & 2.79 & 2.07 & 2.53 & 2.57 & 1.53 & 0.74 & 1.22 & 1.02 & 0.60 \\
\hline Nigeria & 0.27 & 0.21 & 0.05 & 0.27 & 0.33 & 0.80 & 0.24 & 5.16 & 1.24 \\
\hline Norway & 0.41 & 0.41 & 0.28 & 0.29 & 0.38 & 0.98 & 0.69 & 1.04 & 1.30 \\
\hline Panama & 0.04 & 0.04 & 0.03 & 0.05 & 0.10 & 1.04 & 0.74 & 1.87 & 1.95 \\
\hline Philippines & 0.15 & 0.25 & 0.09 & 0.10 & 0.11 & 1.68 & 0.36 & 1.12 & 1.11 \\
\hline Poland & 0.02 & 0.62 & 0.37 & 0.55 & 0.65 & 26.40 & 0.60 & 1.49 & 1.17 \\
\hline
\end{tabular}


8 MUDRA: Journal of Finance and Accounting, Vol. 1, Issue 2

\begin{tabular}{|c|c|c|c|c|c|c|c|c|c|}
\hline \multirow[t]{2}{*}{ Countries } & \multicolumn{5}{|c|}{ FDI - Relative Index } & \multicolumn{4}{|c|}{ FDI - Temporal Index } \\
\hline & 1990 & 1995 & 2000 & 2005 & 2009 & $\begin{array}{r}1995 \\
/ 1990 \\
\end{array}$ & $\begin{array}{l}2000 / \\
1995\end{array}$ & $\begin{array}{l}2005 / \\
2000\end{array}$ & $\begin{array}{l}2009 / \\
2005\end{array}$ \\
\hline Portugal & 0.77 & 0.12 & 0.26 & 0.21 & 0.16 & 0.15 & 2.26 & 0.80 & 0.77 \\
\hline $\begin{array}{l}\text { Russian } \\
\text { Federation }\end{array}$ & 0.00 & 0.35 & 0.11 & 0.69 & 2.20 & 336.29 & 0.31 & 6.45 & 3.17 \\
\hline Seychelles & 0.00 & 0.01 & 0.00 & 0.00 & 0.01 & 110.74 & 0.12 & 4.79 & 2.99 \\
\hline Singapore & 1.48 & 1.94 & 0.65 & 0.77 & 0.95 & 1.31 & 0.34 & 1.18 & 1.24 \\
\hline South Africa & 0.03 & 0.21 & 0.04 & 0.36 & 0.32 & 6.26 & 0.17 & 10.16 & 0.91 \\
\hline South Korea & 0.20 & 0.21 & 0.36 & 0.38 & 0.33 & 1.06 & 1.67 & 1.06 & 0.88 \\
\hline Spain & 3.52 & 1.36 & 1.57 & 1.34 & 0.85 & 0.39 & 1.15 & 0.86 & 0.63 \\
\hline Sri Lanka & 0.01 & 0.01 & 0.01 & 0.01 & 0.02 & 0.95 & 0.63 & 2.14 & 1.57 \\
\hline Sweden & 0.52 & 2.43 & 0.93 & 0.54 & 0.62 & 4.65 & 0.38 & 0.58 & 1.14 \\
\hline Switzerland & 1.45 & 0.37 & 0.76 & 0.85 & 0.55 & 0.26 & 2.04 & 1.12 & 0.64 \\
\hline Thailand & 0.68 & 0.35 & 0.13 & 0.43 & 0.34 & 0.51 & 0.38 & 3.26 & 0.78 \\
\hline Tunisia & 0.02 & 0.06 & 0.03 & 0.04 & 0.10 & 2.70 & 0.48 & 1.36 & 2.28 \\
\hline Turkey & 0.18 & 0.15 & 0.04 & 0.54 & 0.43 & 0.82 & 0.26 & 13.87 & 0.80 \\
\hline United Kingdom & 8.07 & 3.36 & 4.70 & 9.45 & 2.59 & 0.42 & 1.40 & 2.01 & 0.27 \\
\hline United States & 12.83 & 9.89 & $\begin{array}{c}12.4 \\
2\end{array}$ & 5.63 & 7.37 & 0.77 & 1.26 & 0.45 & 1.31 \\
\hline $\begin{array}{l}\text { Venezuela } \\
\text { (Bolivarian } \\
\text { Republic of) }\end{array}$ & 0.21 & 0.17 & 0.19 & 0.14 & 0.16 & 0.80 & 1.12 & 0.75 & 1.14 \\
\hline Average & 1.00 & 1.00 & 1.00 & 1.00 & 1.00 & 11.34 & 1.95 & 2.41 & 1.44 \\
\hline $\begin{array}{l}\text { Change in } \\
\text { Average }\end{array}$ & & & & & & & 0.17 & 1.23 & 0.60 \\
\hline $\begin{array}{l}\text { Standard } \\
\text { Deviation }\end{array}$ & & & & & & 48.16 & 6.44 & 2.52 & 1.16 \\
\hline $\begin{array}{l}\text { Coefficient of } \\
\text { Variation }\end{array}$ & & & & & & 424.53 & 329.67 & 104.81 & 80.27 \\
\hline $\begin{array}{l}\text { Coefficient of } \\
\text { Variation Ratio }\end{array}$ & & & & & & 5.29 & 4.11 & 1.31 & 1.00 \\
\hline
\end{tabular}

In case of absolute amount of GDP, the standard deviation increases significantly with the increase of average GDP. However the $\mathrm{CV}$ and $\mathrm{CV}$ ratio have been continuously decreasing except in 2000. In case of Temporal Index of GDP, the average is almost 
Global Inward Foreign Direct Investment: Trends and Concentration Patterns $\mid 9$

constant with a small change for all this period. However the standard deviation, CV and CV ratio have been continuously decreasing (Table $1 b$ ).

Table 1b: Indices of Country-wise Distribution of GDP

\begin{tabular}{|c|c|c|c|c|c|c|c|c|c|}
\hline \multirow[t]{2}{*}{ Countries } & \multicolumn{5}{|c|}{ GDP - Relative Index } & \multicolumn{4}{|c|}{ GDP - Temporal Index } \\
\hline & 1990 & 1995 & 2000 & 2005 & 2009 & $\begin{array}{c}1995 / \\
1990\end{array}$ & $\begin{array}{l}2000 / \\
1995\end{array}$ & $\begin{array}{l}2005 / \\
2000\end{array}$ & $\begin{array}{l}2009 / \\
2005\end{array}$ \\
\hline Argentina & 0.37 & 0.49 & 0.50 & 0.23 & 0.32 & 1.34 & 1.03 & 0.46 & 1.37 \\
\hline Australia & 0.83 & 0.73 & 0.71 & 0.94 & 0.98 & 0.88 & 0.97 & 1.33 & 1.04 \\
\hline Austria & 0.43 & 0.45 & 0.34 & 0.39 & 0.39 & 1.06 & 0.75 & 1.13 & 1.00 \\
\hline Bahrain & 0.01 & 0.01 & 0.01 & 0.02 & 0.02 & 1.00 & 1.28 & 1.21 & 1.24 \\
\hline $\begin{array}{l}\text { Belgium- } \\
\text { Luxembourg }\end{array}$ & 0.56 & 0.58 & 0.45 & 0.53 & 0.53 & 1.04 & 0.77 & 1.18 & 1.00 \\
\hline Brazil & 1.24 & 1.46 & 1.14 & 1.12 & 1.56 & 1.18 & 0.78 & 0.98 & 1.39 \\
\hline $\begin{array}{l}\text { British Virgin } \\
\text { Islands }\end{array}$ & 0.00 & 0.00 & 0.00 & 0.00 & 0.00 & 2.77 & 1.84 & 0.89 & 1.07 \\
\hline Canada & 1.51 & 1.12 & 1.28 & 1.44 & 1.36 & 0.74 & 1.14 & 1.13 & 0.94 \\
\hline Chile & 0.09 & 0.14 & 0.13 & 0.15 & 0.17 & 1.57 & 0.97 & 1.13 & 1.10 \\
\hline China & 1.05 & 1.44 & 2.11 & 2.93 & 4.78 & 1.37 & 1.47 & 1.39 & 1.63 \\
\hline $\begin{array}{l}\text { China, Hong } \\
\text { Kong SAR }\end{array}$ & 0.20 & 0.27 & 0.30 & 0.23 & 0.21 & 1.37 & 1.09 & 0.76 & 0.95 \\
\hline $\begin{array}{l}\text { China, Taiwan } \\
\text { Province of }\end{array}$ & 0.43 & 0.52 & 0.57 & 0.45 & 0.37 & 1.22 & 1.09 & 0.80 & 0.83 \\
\hline Colombia & 0.12 & 0.18 & 0.15 & 0.16 & 0.23 & 1.42 & 0.84 & 1.06 & 1.49 \\
\hline Costa Rica & 0.02 & 0.02 & 0.03 & 0.03 & 0.03 & 1.18 & 1.27 & 0.90 & 1.17 \\
\hline Czech Republic & 0.14 & 0.10 & 0.10 & 0.16 & 0.20 & 0.75 & 0.96 & 1.58 & 1.26 \\
\hline Denmark & 0.35 & 0.35 & 0.28 & 0.33 & 0.32 & 0.98 & 0.82 & 1.16 & 0.96 \\
\hline Egypt & 0.10 & 0.13 & 0.18 & 0.13 & 0.19 & 1.28 & 1.35 & 0.71 & 1.53 \\
\hline France & 3.23 & 2.98 & 2.35 & 2.72 & 2.72 & 0.92 & 0.79 & 1.16 & 1.00 \\
\hline Germany & 4.45 & 4.79 & 3.36 & 3.55 & 3.40 & 1.08 & 0.70 & 1.06 & 0.96 \\
\hline Hungary & 0.10 & 0.09 & 0.08 & 0.14 & 0.13 & 0.91 & 0.97 & 1.66 & 0.93 \\
\hline India & 0.85 & 0.70 & 0.83 & 1.03 & 1.25 & 0.83 & 1.18 & 1.24 & 1.22 \\
\hline Ireland & 0.12 & 0.13 & 0.17 & 0.26 & 0.24 & 1.03 & 1.34 & 1.50 & 0.92 \\
\hline
\end{tabular}


10 | MUDRA: Journal of Finance and Accounting, Vol. 1, Issue 2

\begin{tabular}{|c|c|c|c|c|c|c|c|c|c|}
\hline \multirow[t]{2}{*}{ Countries } & \multicolumn{5}{|c|}{ GDP - Relative Index } & \multicolumn{4}{|c|}{ GDP - Temporal Index } \\
\hline & 1990 & 1995 & 2000 & 2005 & 2009 & $\begin{array}{c}1995 / \\
1990\end{array}$ & $\begin{array}{c}2000 / \\
1995\end{array}$ & $\begin{array}{c}2005 / \\
2000\end{array}$ & $\begin{array}{l}2009 / \\
2005\end{array}$ \\
\hline Israel & 0.15 & 0.18 & 0.21 & 0.17 & 0.20 & 1.22 & 1.19 & 0.78 & 1.17 \\
\hline Italy & 2.94 & 2.14 & 1.94 & 2.25 & 2.16 & 0.73 & 0.91 & 1.16 & 0.96 \\
\hline Jamaica & 0.01 & 0.01 & 0.01 & 0.01 & 0.01 & 0.99 & 1.27 & 0.89 & 1.05 \\
\hline Japan & 7.84 & 9.96 & 8.26 & 5.79 & 5.18 & 1.27 & 0.83 & 0.70 & 0.89 \\
\hline Lebanon & 0.01 & 0.02 & 0.03 & 0.03 & 0.03 & 2.85 & 1.42 & 0.93 & 1.20 \\
\hline Malaysia & 0.12 & 0.18 & 0.17 & 0.18 & 0.19 & 1.48 & 0.95 & 1.06 & 1.11 \\
\hline Mauritius & 0.01 & 0.01 & 0.01 & 0.01 & 0.01 & 1.14 & 1.06 & 0.99 & 1.14 \\
\hline Mexico & 0.68 & 0.54 & 1.03 & 0.98 & 0.88 & 0.80 & 1.89 & 0.95 & 0.91 \\
\hline Morocco & 0.07 & 0.07 & 0.07 & 0.08 & 0.09 & 0.93 & 0.94 & 1.14 & 1.20 \\
\hline Netherlands & 0.77 & 0.80 & 0.68 & 0.81 & 0.81 & 1.04 & 0.86 & 1.18 & 1.00 \\
\hline Nigeria & 0.09 & 0.06 & 0.08 & 0.14 & 0.19 & 0.63 & 1.43 & 1.74 & 1.30 \\
\hline Norway & 0.31 & 0.28 & 0.30 & 0.38 & 0.39 & 0.93 & 1.05 & 1.29 & 1.02 \\
\hline Panama & 0.02 & 0.02 & 0.02 & 0.02 & 0.03 & 1.09 & 1.20 & 0.96 & 1.27 \\
\hline Philippines & 0.12 & 0.14 & 0.13 & 0.13 & 0.16 & 1.22 & 0.95 & 0.94 & 1.31 \\
\hline Poland & 0.17 & 0.26 & 0.30 & 0.39 & 0.44 & 1.58 & 1.15 & 1.28 & 1.13 \\
\hline Portugal & 0.20 & 0.21 & 0.20 & 0.24 & 0.23 & 1.10 & 0.93 & 1.18 & 0.98 \\
\hline $\begin{array}{l}\text { Russian } \\
\text { Federation }\end{array}$ & 1.23 & 0.76 & 0.46 & 0.97 & 1.26 & 0.62 & 0.61 & 2.12 & 1.30 \\
\hline Seychelles & 0.00 & 0.00 & 0.00 & 0.00 & 0.00 & 1.01 & 1.13 & 0.84 & 0.85 \\
\hline Singapore & 0.10 & 0.16 & 0.16 & 0.15 & 0.17 & 1.66 & 1.03 & 0.93 & 1.14 \\
\hline South Africa & 0.29 & 0.29 & 0.24 & 0.31 & 0.29 & 0.99 & 0.82 & 1.31 & 0.94 \\
\hline South Korea & 0.68 & 0.98 & 0.91 & 1.01 & 0.85 & 1.43 & 0.92 & 1.11 & 0.84 \\
\hline Spain & 1.35 & 1.13 & 1.03 & 1.44 & 1.50 & 0.84 & 0.91 & 1.40 & 1.04 \\
\hline Sri Lanka & 0.02 & 0.03 & 0.03 & 0.03 & 0.04 & 1.19 & 1.17 & 1.04 & 1.41 \\
\hline Sweden & 0.64 & 0.48 & 0.43 & 0.47 & 0.41 & 0.76 & 0.90 & 1.07 & 0.88 \\
\hline Switzerland & 0.62 & 0.60 & 0.45 & 0.48 & 0.50 & 0.97 & 0.74 & 1.07 & 1.04 \\
\hline Thailand & 0.22 & 0.32 & 0.22 & 0.22 & 0.28 & 1.44 & 0.68 & 1.03 & 1.24 \\
\hline Tunisia & 0.03 & 0.03 & 0.03 & 0.04 & 0.04 & 1.07 & 1.00 & 1.07 & 1.07 \\
\hline Turkey & 0.39 & 0.32 & 0.35 & 0.46 & 0.64 & 0.82 & 1.10 & 1.31 & 1.38 \\
\hline
\end{tabular}




\begin{tabular}{|c|c|c|c|c|c|c|c|c|c|}
\hline \multirow[t]{2}{*}{ Countries } & \multicolumn{5}{|c|}{ GDP - Relative Index } & \multicolumn{4}{|c|}{ GDP - Temporal Index } \\
\hline & 1990 & 1995 & 2000 & 2005 & 2009 & $\begin{array}{c}1995 / \\
1990\end{array}$ & $\begin{array}{l}2000 / \\
1995\end{array}$ & $\begin{array}{l}2005 / \\
2000\end{array}$ & $\begin{array}{l}2009 / \\
2005\end{array}$ \\
\hline United Kingdom & 2.59 & 2.17 & 2.57 & 2.86 & 2.22 & 0.84 & 1.18 & 1.11 & 0.78 \\
\hline United States & 15.03 & 14.03 & 17.40 & 15.86 & 14.05 & 0.93 & 1.24 & 0.91 & 0.89 \\
\hline $\begin{array}{l}\text { Venezuela } \\
\text { (Bolivarian } \\
\text { Republic of) }\end{array}$ & 0.12 & 0.14 & 0.21 & 0.19 & 0.34 & 1.16 & 1.46 & 0.89 & 1.86 \\
\hline Average & 1.00 & 1.00 & 1.00 & 1.00 & 1.00 & 1.14 & 1.06 & 1.11 & 1.12 \\
\hline $\begin{array}{l}\text { Change in } \\
\text { Average }\end{array}$ & & & & & & & 0.93 & 1.04 & 1.01 \\
\hline $\begin{array}{l}\text { Standard } \\
\text { Deviation }\end{array}$ & & & & & & 0.41 & 0.27 & 0.28 & 0.22 \\
\hline $\begin{array}{l}\text { Coefficient of } \\
\text { Variation }\end{array}$ & & & & & & 36.26 & 25.00 & 25.19 & 19.67 \\
\hline $\begin{array}{l}\text { Coefficient of } \\
\text { Variation Ratio }\end{array}$ & & & & & & 1.84 & 1.27 & 1.28 & 1.00 \\
\hline
\end{tabular}

From above analysis we are able to find out countries which are attracting relatively more FDI inflows with increase in GDP and countries which are attracting relatively less FDI inflows with increase in GDP. Next we study distribution pattern of FDI. Our hypothesis is that countries are attracting proportionate share of FDI with respect to GDP. It means higher GDP country attracts higher FDI and low GDP country attracts low FDI. Scale of measure of this hypothesis is as:

- If Ratio of Temporal Index of FDI \& GDP $>$ One $\rightarrow$ More elastic

- If Ratio of Temporal Index of FDI \& GDP $=$ One $\rightarrow$ Unit elasticity

- If Ratio of Temporal Index of FDI \& GDP $<$ One $\rightarrow$ Inelastic elastic

Ratio of temporal indices of FDI and GDP indicates FDI inflows with respect to GDP at two different point of time. Estimated average of these indices in 1995/1900, 2000/1995, 2005/2000 and 2009/2005 are 14.68, 2.11, 2.13 and 1.33 respectively (Table 1c). Elasticity is falling. It indicates the initial advantages of liberalisation and globalisation is going down. It means on an average the countries attracting FDI greater than their due share. It means that some countries are attracting more FDI than their proportionate share with respect to GDP. GDP of a country indicates general growth. Therefore countries which have higher GDP growth should also have higher FDI growth. But the analysis shows that distribution of FDI across countries is not made on the basis of GDP growth. However average FDI has been continuously decreasing over different point of time. This implies 
12 MUDRA: Journal of Finance and Accounting, Vol. 1, Issue 2

that concentration of FDI has been decreasing with the increase of competition for FDI among countries.

Table 1c: Ratio of Temporal Index of FDI and GDP

\begin{tabular}{|l|c|c|c|c|}
\hline \multirow{2}{*}{ Countries } & \multicolumn{4}{c|}{ Ratio of Temporal Index of FDI \& GDP } \\
\cline { 2 - 5 } & $\mathbf{1 9 9 5 / 1 9 9 0}$ & $\mathbf{2 0 0 0 / 1 9 9 5}$ & $\mathbf{2 0 0 5 / 2 0 0 0}$ & $\mathbf{2 0 0 9 / 2 0 0 5}$ \\
\hline Argentina & 1.45 & 0.43 & 1.48 & 0.72 \\
\hline Australia & 1.06 & 0.28 & 2.38 & 0.70 \\
\hline Austria & 1.74 & 1.45 & 1.46 & 0.69 \\
\hline Bahrain & 0.74 & 0.16 & 3.25 & 0.21 \\
\hline Belgium-Luxembourg & 0.81 & 2.53 & 0.52 & 1.59 \\
\hline Brazil & 2.41 & 2.24 & 0.63 & 1.31 \\
\hline British Virgin Islands & 11.17 & 1.41 & 1.88 & 2.05 \\
\hline Canada & 1.05 & 1.48 & 0.46 & 0.81 \\
\hline Chile & 1.80 & 0.40 & 1.72 & 1.75 \\
\hline China & 4.99 & 0.17 & 1.74 & 0.85 \\
\hline China, Hong Kong SAR & 0.88 & 2.14 & 0.97 & 1.60 \\
\hline China, Taiwan Province of & 0.61 & 0.68 & 0.56 & 2.20 \\
\hline Colombia & 0.87 & 0.70 & 5.41 & 0.50 \\
\hline Costa Rica & 1.12 & 0.22 & 3.18 & 1.38 \\
\hline Czech Republic & 13.08 & 0.48 & 2.00 & 0.20 \\
\hline Denmark & 2.48 & 1.09 & 0.63 & 0.96 \\
\hline Egypt & 0.40 & 0.36 & 8.32 & 0.86 \\
\hline France & 1.04 & 0.54 & 2.30 & 0.74 \\
\hline Germany & 2.40 & 5.52 & 0.31 & 0.83 \\
\hline Hungary & 6.41 & 0.13 & 2.28 & 1.02 \\
\hline India & 6.98 & 0.33 & 2.32 & 3.95 \\
\hline Ireland & 1.44 & 3.14 & 0.60 & 1.68 \\
\hline Israel & 6.01 & 0.74 & 1.25 & 0.82 \\
\hline
\end{tabular}




\begin{tabular}{|l|c|c|c|c|}
\hline \multirow{2}{*}{ Countries } & \multicolumn{4}{c|}{ Ratio of Temporal Index of FDI \& GDP } \\
\cline { 2 - 5 } & $\mathbf{1 9 9 5 / 1 9 9 0}$ & $\mathbf{2 0 0 0 / 1 9 9 5}$ & $\mathbf{2 0 0 5 / 2 0 0 0}$ & $\mathbf{2 0 0 9 / 2 0 0 5}$ \\
\hline Italy & 0.66 & 0.72 & 1.75 & 1.69 \\
\hline Jamaica & 0.54 & 0.59 & 2.23 & 1.56 \\
\hline Japan & 0.01 & 56.86 & 0.65 & 5.08 \\
\hline Lebanon & 1.21 & 4.57 & 3.97 & 1.61 \\
\hline Malaysia & 0.96 & 0.16 & 1.38 & 0.32 \\
\hline Mauritius & 0.25 & 3.29 & 0.21 & 5.74 \\
\hline Mexico & 2.88 & 0.24 & 1.74 & 0.67 \\
\hline Morocco & 1.37 & 0.32 & 4.65 & 0.71 \\
\hline Netherlands & 0.71 & 1.42 & 0.86 & 0.60 \\
\hline Nigeria & 1.27 & 0.17 & 2.96 & 0.95 \\
\hline Norway & 1.06 & 0.66 & 0.80 & 1.28 \\
\hline Panama & 0.96 & 0.62 & 1.95 & 1.53 \\
\hline Philippines & 1.38 & 0.38 & 1.20 & 0.85 \\
\hline Poland & 16.76 & 0.52 & 1.17 & 1.04 \\
\hline Portugal & 0.14 & 2.43 & 0.68 & 0.79 \\
\hline Russian Federation & 545.80 & 0.51 & 3.04 & 2.44 \\
\hline Seychelles & 109.84 & 0.11 & 5.70 & 3.53 \\
\hline Singapore & 0.79 & 0.33 & 1.27 & 1.08 \\
\hline South Africa & 6.35 & 0.21 & 7.74 & 0.96 \\
\hline South Korea & 0.74 & 1.81 & 0.96 & 1.04 \\
\hline Spain & 0.46 & 1.27 & 0.61 & 0.61 \\
\hline Sri Lanka & 0.80 & 0.54 & 2.04 & 1.11 \\
\hline Sweden & 6.14 & 0.42 & 0.55 & 1.29 \\
\hline Switzerland & 0.26 & 2.76 & 1.05 & 0.62 \\
\hline Thailand & 0.35 & 0.56 & 3.15 & 0.63 \\
\hline Tunisia & 2.52 & 0.48 & 1.27 & 2.13 \\
\hline Turkey & 1.00 & 0.24 & 10.59 & 0.58 \\
\hline
\end{tabular}


14 | MUDRA: Journal of Finance and Accounting, Vol. 1, Issue 2

\begin{tabular}{|l|c|c|c|c|}
\hline \multirow{2}{*}{ Countries } & \multicolumn{4}{c|}{ Ratio of Temporal Index of FDI \& GDP } \\
\cline { 2 - 5 } & $\mathbf{1 9 9 5 / 1 9 9 0}$ & $\mathbf{2 0 0 0 / 1 9 9 5}$ & $\mathbf{2 0 0 5 / 2 0 0 0}$ & $\mathbf{2 0 0 9 / 2 0 0 5}$ \\
\hline United Kingdom & 0.50 & 1.18 & 1.81 & 0.35 \\
\hline United States & 0.83 & 1.01 & 0.50 & 1.48 \\
\hline $\begin{array}{l}\text { Venezuela } \\
\text { (Bolivarian Republic of) }\end{array}$ & 0.69 & 0.77 & 0.84 & 0.61 \\
\hline Average & 14.68 & 2.11 & 2.13 & 1.33 \\
\hline Change in Average & & 0.14 & 1.01 & 0.62 \\
\hline Standard Deviation & & & & \\
\hline Coefficient of Variation & & & & \\
\hline Coefficient of Variation Ratio & & & & \\
\hline
\end{tabular}

\subsection{Concentration of FDI Inflows and Stock}

With globalisation and liberalisation, the international FDI should be uniformly distributed to enable a rational distribution of resources. It implies that the concentration of inward FDI should be low amongst the recipient (host) countries.

Herfindal's index of concentration clearly indicates that there is a low concentration in distribution of FDI in terms of inflow and stock. The maximum value of concentration is 0.111 in 1999 in case of FDI inflow (Table 2). This value indicates that concentration is low in case of distribution of FDI inflow. It means that competition amongst countries for attraction of FDI inflow in their respective countries is increasing over a period of time. Due to low concentration and global competition, share of developing countries has crossed more than 40 percent in terms of inflow. In terms of FDI stock, the concentration ratio is also low. It implies that distribution of FDI has been moving towards uniform distribution of productive resources across the countries, which is a basic purpose of globalisation and competition. The low concentration ratio implies that the share of developing countries is increasing because earlier developed countries were main recipient of FDI. And FDI mainly concentrated in a few developed countries only.

Figure 1 gives pictorial view of concentration ratio in terms of flow and stock. The concentration ratio is declining over periods in both flow and stock terms. There is a high fluctuation in FDI inflow. This indicates concentration of FDI inflow is decreasing and increasing over periods. In net concentration of FDI inflow has decreased from 0.104 in 1990 to 0.078 in 2009 (Table 2). The line of concentration ratio of FDI stock shows a more consistent trend than inflow. The concentration ratio of stock is consistently decreasing 
from 0.090 in 1990 to 0.073 in 2009 (Table 2). It implies that world productive resources are shifting from developed countries to developing countries on a permanent basis.

Table 2: Herfindal's Index of Concentration of FDI during 1990-2009

\begin{tabular}{|c|c|c|}
\hline Year & FDI Inflows & FDI Inward Stocks \\
\hline 1990 & 0.104 & 0.090 \\
\hline 1991 & 0.068 & 0.086 \\
\hline 1992 & 0.059 & 0.081 \\
\hline 1993 & 0.099 & 0.081 \\
\hline 1994 & 0.077 & 0.075 \\
\hline 1995 & 0.073 & 0.073 \\
\hline 1996 & 0.089 & 0.072 \\
\hline 1997 & 0.083 & 0.075 \\
\hline 1998 & 0.104 & 0.072 \\
\hline 1999 & 0.111 & 0.075 \\
\hline 2000 & 0.101 & 0.084 \\
\hline 2001 & 0.081 & 0.084 \\
\hline 2002 & 0.061 & 0.074 \\
\hline 2003 & 0.054 & 0.065 \\
\hline 2004 & 0.069 & 0.062 \\
\hline 2005 & 0.069 & 0.064 \\
\hline 2006 & 0.069 & 0.061 \\
\hline 2007 & 0.064 & 0.057 \\
\hline 2008 & 0.073 & 0.060 \\
\hline 2009 & 0.055 & 0.067 \\
\hline Average & 0.078 & 0.073 \\
\hline
\end{tabular}

Regression statistics tell us the rate at which concentration of FDI is decreasing. In case of FDI inflow, growth rate is 1.7 percent, negative and statistically significant at 5 percent. It clearly indicates that concentration of FDI inflow is decreasing over periods. Regression statistics of FDI inward stock follow pattern of inflow. Growth rate is 1.8 percent, negative and highly significant at one percent (Table 3). Concentration of FDI has decreased on an overall basis. 
Figure 1: Concentration of FDI Inflows and Inward Stock during 1990-2009

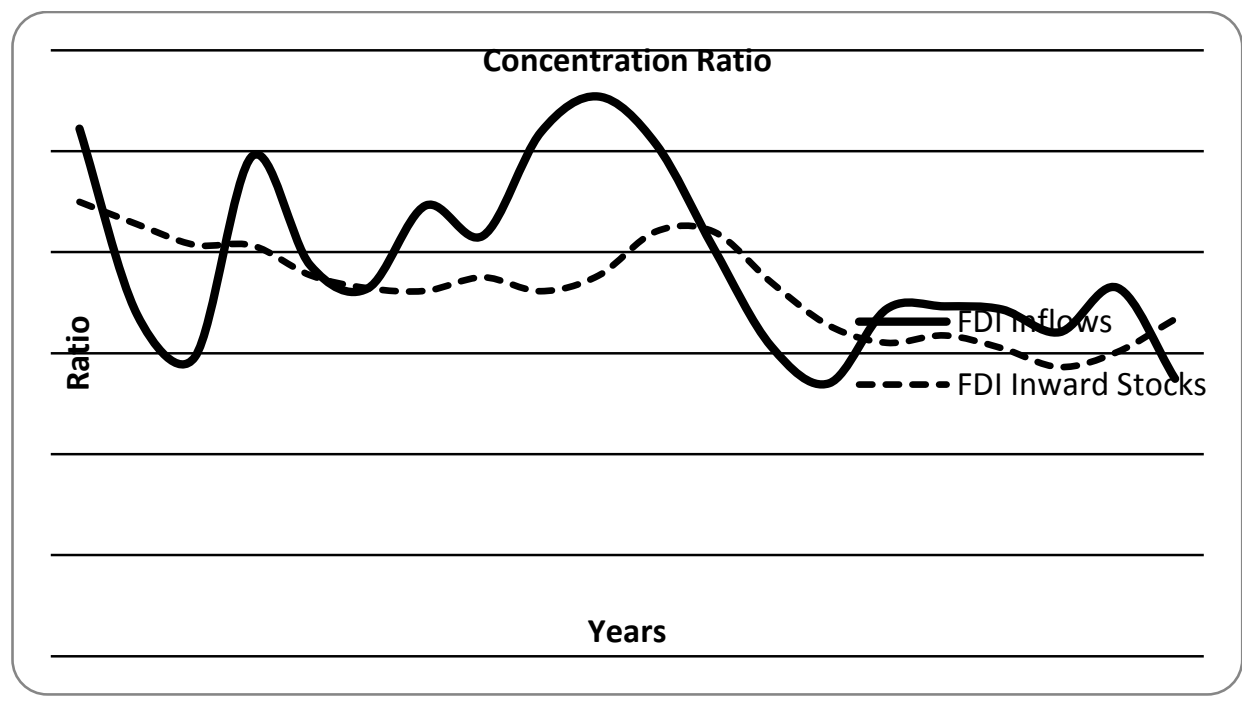

Table 3: Regression Statistics of Herfindal's Index of Concentration during1990-2009

\begin{tabular}{|l|c|c|}
\hline & FDI Inflows & FDI Inward Stocks \\
\hline Adjusted R Sq & 0.152 & 0.653 \\
\hline Coefficient & -0.017 & -0.018 \\
\hline $\mathrm{p}$ value & 0.050 & 0.000 \\
\hline
\end{tabular}

Now we examine the concentration of FDI on country grouping basis- developed and developing countries. In case of FDI inflow, the concentration ratio of developed countries is 0.128 on an average basis during 1990-2009 which is slightly higher than the concentration ratio of developing countries that is 0.111 (Table 4). It implies that FDI inflow is more concentrated in a few developed countries that that of developing countries. It means that developing countries are more competitive than developed countries. On a net basis, the concentration ratio is low in both developed and developing countries. Competition is increasing among developed and developing countries for FDI across countries. In case of FDI inward stock, the concentration ratio of developed countries is very slightly higher than that of developing countries on an average basis during 19902009. However, during pre-WTO period, the concentration ratio of stock is high in case of developed and developing countries. Gradually this ratio is decreasing. It implies that during pre-WTO periods, FDI stock was concentrated in a few developed and developing 
countries. After WTO global competitive environment improved, this led to an increase in competition for FDI across countries. Finally, this helps in decreasing concentration of FDI in a few countries and leads to uniform distribution of global productive resources which increases global welfare.

Table 4: Herfindal's Index of Concentration of FDI of Developed and Developing Countries during 1990-2009

\begin{tabular}{|c|c|c|c|c|}
\hline \multirow{2}{*}{ Year } & \multicolumn{2}{|c|}{ FDI:Inflows } & \multicolumn{2}{c|}{ FDI:Inward Stock } \\
\cline { 2 - 5 } & Developed & Developing & Developed & Developing \\
\hline 1990 & 0.141 & 0.082 & 0.131 & $\mathbf{0 . 1 9 7}$ \\
\hline 1991 & 0.102 & 0.088 & 0.126 & 0.176 \\
\hline 1992 & 0.097 & 0.096 & 0.124 & 0.156 \\
\hline 1993 & 0.170 & $\mathbf{0 . 1 8 2}$ & 0.129 & 0.141 \\
\hline 1994 & 0.130 & 0.171 & 0.119 & 0.133 \\
\hline 1995 & 0.118 & 0.161 & 0.115 & 0.123 \\
\hline 1996 & 0.169 & 0.132 & 0.118 & 0.112 \\
\hline 1997 & $\mathbf{0 . 1 7 1}$ & 0.103 & 0.128 & 0.102 \\
\hline 1998 & 0.164 & 0.115 & 0.120 & 0.088 \\
\hline 1999 & 0.164 & 0.093 & 0.128 & 0.113 \\
\hline 2000 & 0.141 & 0.119 & 0.141 & 0.114 \\
\hline 2001 & 0.127 & 0.109 & $\mathbf{0 . 1 4 2}$ & 0.101 \\
\hline 2002 & 0.087 & 0.147 & 0.117 & 0.087 \\
\hline 2003 & 0.080 & 0.126 & 0.101 & 0.083 \\
\hline 2004 & 0.124 & 0.094 & 0.094 & 0.082 \\
\hline 2005 & 0.113 & 0.086 & 0.102 & 0.078 \\
\hline 2006 & 0.113 & 0.070 & 0.097 & 0.085 \\
\hline 2007 & 0.101 & 0.073 & 0.091 & 0.102 \\
\hline 2008 & 0.139 & 0.080 & 0.097 & 0.078 \\
\hline 2009 & 0.099 & 0.087 & 0.107 & 0.076 \\
\hline Average & 0.128 & 0.111 & 0.116 & 0.111 \\
\hline
\end{tabular}

Figure 2 shows that the patterns in concentration of flows are divergent, as between developed and developing countries. This trend points towards a substitution between, the two broad groups of countries, in terms of concentration of capital flows. It means when concentration of FDI inflow is decreasing in developed countries then it leads to increase 
18 | MUDRA: Journal of Finance and Accounting, Vol. 1, Issue 2

in developing countries. In terms of FDI stock, concentration of stock is consistently decreasing in both developed and developing countries. Decreasing rate is high in case of developing countries (Figure 3).

Figure 2: Concentration of FDI Inflows of Developed and Developing Countries

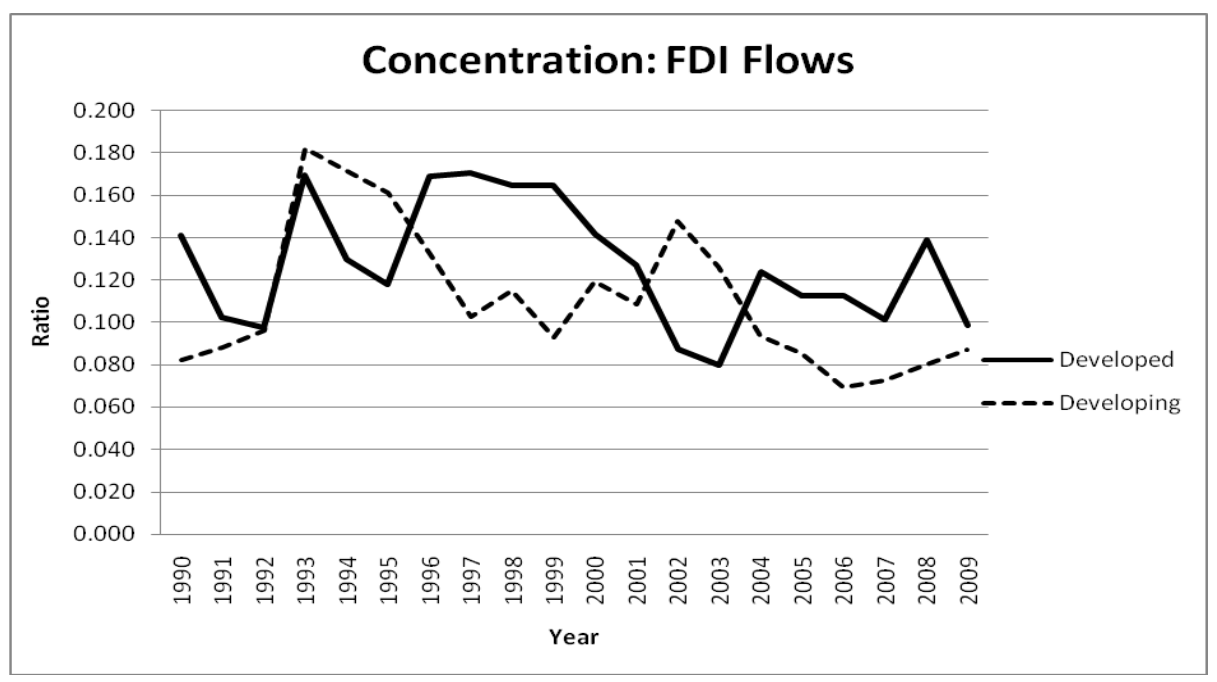

Figure 3: Concentration of FDI Inward Stock of Developed and Developing Countries

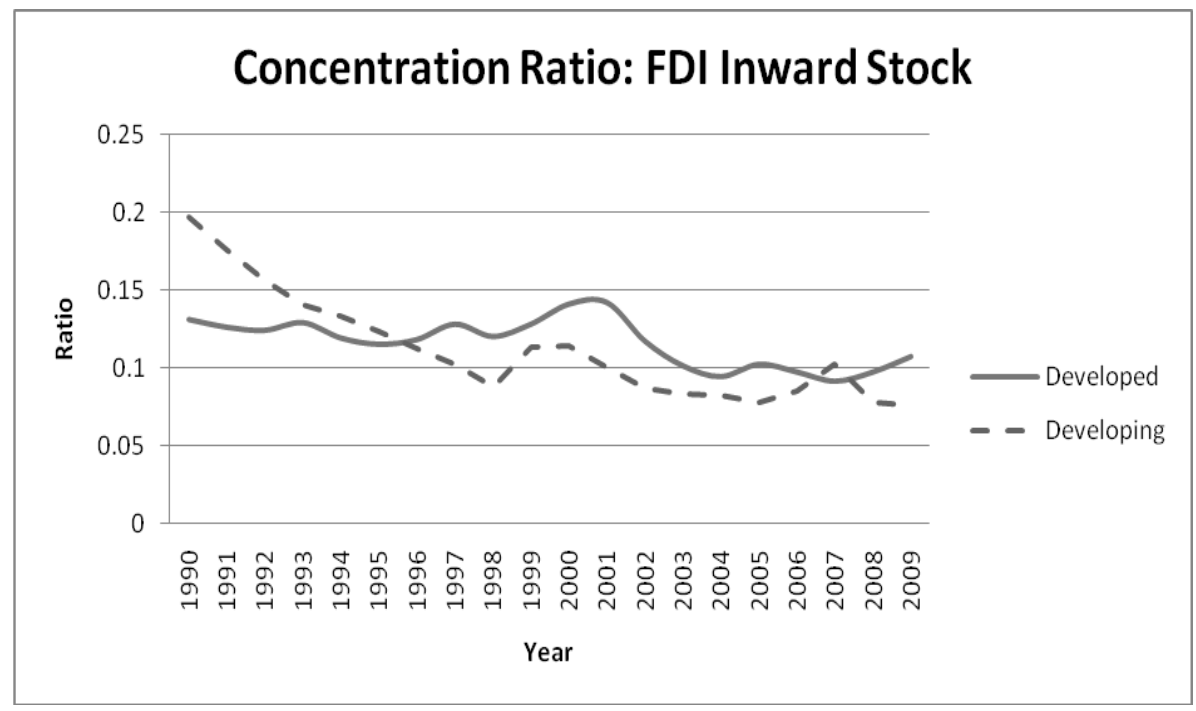


The first observation is that inward capital movements as measured by flows and stock generally show a decline in the concentration ratio. Regression statistics suggests that on three out of four counts the global economy has experienced declining concentration ratios. The coefficient is negative in all four cases and significant except in case of developed countries. This means that except for inflows in the case of developed countries (which they largely receive from developed countries) the distribution of capital flows is more uniform across countries. This should augur well for the global economy. Since our interest lies with developing economies it shows that developing economies gain significantly in both terms - stock and flow $(-0.044$ and -0.021$)$ (Table 5). The gain is greater in stock, which points to a more uniform factor endowment of capital emerging in developing countries. A caveat to this trend may be whether the developing countries that are beneficiaries of the spreading out are able to utilize these capital stocks better and further attract FDI.

Table 5: Regression Statistics of Herfindal's Index of Concentration during 1990-2009

\begin{tabular}{|l|c|c|c|c|}
\hline \multirow{2}{*}{} & \multicolumn{2}{|c|}{ FDI: Inflows } & \multicolumn{2}{c|}{ FDI: Inward Stock } \\
\cline { 2 - 5 } & Developed & Developing & Developed & Developing \\
\hline Adjusted R Sq. & 0.055 & 0.151 & 0.451 & 0.796 \\
\hline Coefficient & -0.013 & -0.021 & -0.016 & -0.043 \\
\hline P value & 0.164 & 0.051 & 0.001 & 0.000 \\
\hline
\end{tabular}

\subsection{Hypotheses testing}

Hypothesis I: Concentration pattern of inward FDI has not changed.

This hypothesis has been rejected because the concentration of FDI has fallen in terms of inflows and inward stock. The concentration of inward FDI has also fallen in case of developed and developing countries grouping.

Hypothesis II: Concentration pattern of inward FDI has not changed in developing countries.

Concentration of inward FDI has fallen in developing countries. Concentration of FDI inflows and inward stock has fallen at 2.1 percent and 4.3 percent per annum respectively. Therefore, we have rejected this hypothesis.

Hypothesis III: Concentration pattern of inward FDI has not changed in developed countries. 
In case of developed countries, concentration of inward FDI has fallen. Concentration of FDI inflow has fallen at 1.3 percent per annum but not statistically significant. However, concentration of FDI inward stock has decreased at 1.6 percent per annum and it is statistically significant. Therefore, we have rejected this hypothesis.

\subsection{Conclusion}

The purpose of globalisation is that the international FDI would be uniformly distributed to enable a rational and efficient distribution of resources. This could happen only if the concentration of FDI is low or FDI is well distributed. It is therefore apparent that there is a strong relationship between international FDI and concentration pattern.

Since, the ratio of temporal index is high in certain countries; it shows that some of these countries are tax havens which have disproportionate FDI flows in comparison to their GDP. On an average the countries are attracting FDI greater than their due share. Some countries are attracting more FDI than their proportionate share with respect to GDP. GDP of a country indicates general growth. Therefore countries which have higher GDP growth should also have higher FDI growth. But the analysis shows that distribution of FDI across countries is not made on the basis of GDP growth. However average FDI has been continuously decreasing over different point of time. This implies that concentration of FDI inflows has been decreasing with the increase of competition for FDI among countries.

In absolute terms, the concentration of FDI inflow and stock has consistently declined over periods. Due to low concentration and global competition, share of developing countries has crossed more than 40 percent in terms of inflow. Herfindal's index of concentration clearly indicates that there is a low concentration in distribution of FDI in terms of inflow and stock. Concentration of FDI is decreasing at rate of 1.7 percent annual. And it increases competition for FDI inflows. It implies that distribution of FDI has been moving towards uniform distribution of productive resources across the countries, which is a basic purpose of globalisation and competition. The low concentration ratio implies that the share of developing countries is increasing because earlier developed countries were the main recipients of FDI. Also, FDI was mainly concentrated in a few developed countries only. The patterns in concentration of flows are divergent, as between developed and developing countries. This trend points towards a substitution between, the two broad groups of countries, in terms of concentration of capital flows. It means when concentration of FDI inflow is decreasing in developed countries then it leads to increase in developing countries. Regression statistics suggests that on three out of four counts the global economy has experienced declining concentration ratios. The coefficient is negative 
in all four cases and significant except in case of developed countries. This means that except for inflows in the case of developed countries (which they largely receive from developed countries) the distribution of capital flows is more uniform across countries. This should augur well for the global economy. Since our interest lies with developing economies, it shows that developing economies gain significantly in both terms - stock and flow (-0.044 and -0.021). The gain is greater in stock, which points to a more uniform factor endowment of capital emerging in developing countries. A caveat to this trend may be whether the developing countries that are beneficiaries of the spreading out are able to utilize these capital stocks better and further attract FDI. Concentration of inward FDI has decreased on overall basis.

\section{References}

Banga, Rasmi (2006). Do Investment Agreements matter? Journal of Economic Integration, 21(1): pp. 40-63.

Bhanumurthy, K. V. and Deb, A.T. (2008). The determinants and trends of the dynamics of market concentration: The case of deregulating private banking industry in India' SSRN working paper, http://ssrn.com/abstract $=1303418$

Goldar, B. and Ishigami, E. (1999). Foreign Direct Investment in Asia. Economic and Political Weekly, 34(22): M50-M60.

Jha, R. and Bhanu Murthy, K.V. et al (1999). Real consumption levels and public distribution in India. April 10-16, Economic and Political Weekly, Mumbai.

Stallings, Barbara (2007). The globalization of capital flows: Who benefits? American Academy of Political and Social Science, 61: 202-216.

UNCTAD ‘World Investment Report’ various issues, Geneva.

\section{Websites}

www.unctad.org

www.ssrn.com 